International Journal of Modern Physics A

(C) World Scientific Publishing Company

\title{
Effective Abelian-Higgs Theory from SU(2) gauge field theory
}

\author{
VLADIMIR DZHUNUSHALIEV \\ Department of Physics and Microelectronics Engineering, Kyrgyz-Russian Slavic University, \\ Bishkek, Kievskaya Str. 44, 720000, Kyrgyz Republic \\ and \\ Freie Universität Berlin, Fachbereich Physik, Arnimalleee 14, 14195 Berlin, Germany \\ dzhun@hotmail.kg \\ DOUGLAS SINGLETON and DANNY DHOKARH \\ Physics Department, CSU Fresno, 2345 East San Ramon Ave. \\ $M / S 37$ Fresno CA 93740-8031, USA \\ dougs@csufresno.edu ; dannyx@csufresno.edu \\ Received (Day Month Year) \\ Revised (Day Month Year)
}

\begin{abstract}
In the present work we show that it is possible to arrive at a Ginzburg-Landau (GL) like equation from pure $\mathrm{SU}(2)$ gauge theory. This has a connection to the dual superconducting model for color confinement where color flux tubes permanently bind quarks into color neutral states. The GL Lagrangian with a spontaneous symmetry breaking potential, has such (Nielsen-Olesen) flux tube solutions. The spontaneous symmetry breaking requires a tachyonic mass for the effective scalar field. Such a tachyonic mass term is obtained from the condensation of ghost fields.
\end{abstract}

Keywords: Dual superconductivity; confinement; Abelian projection.

\section{Introduction}

In ref. 1 it was shown that one could obtain London's equation from SU(2) gauge theory using the ideas of Abelian projection. This supports the dual superconductor picture of a confining Yang-Mills gauge theory, since London's equation gives a phenomenological description of the Meissner effect. In 1 the quantized $\mathrm{SU}(2)$ gauge fields split into two phases: an ordered and a disordered phase. The ordered phase was the gauge field belonging to the $\mathrm{U}(1)$ subgroup (the diagonal, Abelian component of the $\mathrm{SU}(2)$ gauge field) and the disordered phase was the coset $\mathrm{SU}(2) / \mathrm{SU}(1)$ (the off-diagonal components of the $\mathrm{SU}(2)$ gauge field). The disordered phase played a role similar to the complex scalar field of the GL equation. In 1 we simply set this scalar field to a constant vacuum expectation value i.e. we froze the equations of the $\mathrm{SU}(2) / \mathrm{U}(1)$ fields making them nondynamical degrees of freedom. In this way we obtained London's equation. In the present work we want to "unfreeze" the equations connected with the $\mathrm{SU}(2) / \mathrm{U}(1)$ part, and show that it is possible to 
obtain a GL-like equation. This helps strengthen the dual superconductor picture of the confining Yang-Mills vacuum. The GL Lagrangian density is

$$
\mathcal{L}=-\frac{1}{4} f_{\mu \nu} f^{\mu \nu}+\left(D_{\mu} \varphi^{*}\right)\left(D^{\mu} \varphi\right)-m^{2}|\varphi|^{2}-\lambda|\varphi|^{4}
$$

where $\varphi$ is a complex scalar field, $f_{\mu \nu}=\partial_{\mu} a_{\nu}-\partial_{\nu} a_{\mu}$, is the field strength tensor of the Abelian field, $a_{\mu}$, and $D_{\mu}=\partial_{\mu}-i e a_{\mu}$ is the covariant derivative.

For the dual superconducting picture it is important that the potential for the scalar field in (11) be of the spontaneous symmetry breaking form, and have NielsenOlesen flux tube solutions 2]. This means that the mass term in (11) should be tachyonic $\left(\right.$ i.e. $\left.m^{2}<0\right)$. In this work the tachyonic mass term is generated via the condensation of ghosts as in ref. $\frac{3}{3}$ (see also ref. 4 ).

Strictly the dual superconducting picture of the Yang-Mills vacuum requires a dual GL Lagrangian which gives a dual Meissner effect with respect to the "electric" charges of the theory. In the present work we arrive at the ordinary GL Lagrangian which gives the ordinary Meissner effect with respect to the "magnetic" charges of the theory. However, it is simple to perform the construction in this paper starting with the dual gauge fields and arrive at an effective dual GL Lagrangian. A discussion of dual symmetry and dual gauge fields for Yang-Mills theory can be found in

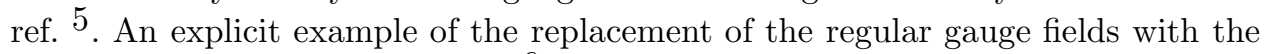
dual gauge fields can be found in $\underline{6}$.

\section{Ordered and disordered phases}

In this section we review the Abelian projection decomposition of the $\mathrm{SU}(2)$ gauge field ${ }^{17}$ and necessary results from ${ }^{1}$. The $\mathrm{SU}(2)$ gauge fields, $\mathcal{A}_{\mu}=\mathcal{A}_{\mu}^{B} T^{B}$, and field strength tensor, $\mathcal{F}_{\mu \nu}^{B}$, can be decomposed as

$$
\begin{aligned}
\mathcal{A}_{\mu} & =\mathcal{A}_{\mu}^{B} T^{B}=a_{\mu} T^{a}+A_{\mu}^{m} T^{m} \\
a_{\mu} & \in U(1) \quad \text { and } A_{\mu}^{m} \in S U(2) / U(1) \\
\mathcal{F}_{\mu \nu}^{B} T^{B} & =\mathcal{F}_{\mu \nu}^{3} T^{3}+\mathcal{F}_{\mu \nu}^{m} T^{m}
\end{aligned}
$$

where

$$
\begin{aligned}
\mathcal{F}_{\mu \nu} & =f_{\mu \nu}+\Phi_{\mu \nu} \in U(1), \quad \mathcal{F}_{\mu \nu}^{m}=F_{\mu \nu}^{m}+G_{\mu \nu}^{m} \in S U(2) / U(1), \\
f_{\mu \nu} & =\partial_{\mu} a_{\nu}-\partial_{\nu} a_{\mu} \text { and } \Phi_{\mu \nu}=g \epsilon^{3 m n} A_{\mu}^{m} A_{\nu}^{n} \in U(1), \\
F_{\mu \nu}^{m} & =\partial_{\mu} A_{\nu}^{m}-\partial_{\nu} A_{\mu}^{m} \text { and } G_{\mu \nu}^{m}=g \epsilon^{3 m n}\left(A_{\mu}^{n} a_{\nu}-A_{\nu}^{n} a_{\mu}\right) \in S U(2) / U(1),
\end{aligned}
$$

where $\epsilon^{A B C}$ are the structural constants of SU(2), $g$ is the coupling constant, $a=3$ is the index of the Abelian subgroup, and $m, n=1,2$ are the indices of the coset. After performing the above decomposition we next apply a quantization technique of Heisenberg ${ }^{[8}$ where the classical fields were replaced by operators $\left(a_{\mu} \rightarrow \hat{a}_{\mu}\right.$ and $A_{\mu}^{m} \rightarrow \hat{A}_{\mu}^{m}$ ) and we then consider expectation values of the fields. This is similar to the field correlators approach in QCD (for review, see 9.) and in ref. 10] similar ideas were used to obtain a set of self coupled equations for the field correlators.

As in 11 we make the following two assumptions 
(1) After quantization the components $\hat{A}_{\mu}^{m}(x)$ become stochastic. In mathematical terms this assumption means that the expectation values of the fields obey

$$
\left\langle A_{\mu}^{m}(x)\right\rangle=0 \quad \text { and } \quad\left\langle A_{\mu}^{m}(x) A_{\nu}^{n}(x)\right\rangle \neq 0
$$

Later we will give a specific form for the nonzero term.

(2) The gauge potentials $a_{\mu}$ and $A_{\mu}^{m}$ are not correlated, and $a_{\mu}$ behaves in a classical manner. Mathematically this means that

$$
\left\langle f\left(a_{\mu}\right) g\left(A_{\nu}^{m}\right)\right\rangle=\left\langle f\left(a_{\mu}\right)\right\rangle\left\langle g\left(A_{\mu}^{m}\right)\right\rangle=f\left(a_{\mu}\right)\left\langle g\left(A_{\mu}^{m}\right)\right\rangle
$$

where $f, g$ are any functions of $a_{\mu}$ and $A_{\mu}^{m}$ respectively. The classical behavior of $a_{\mu}$ results in $\left\langle f\left(a_{\mu}\right)\right\rangle \rightarrow f\left(a_{\mu}\right)$.

In distinction from ref. 1 we will work with the Lagrange density rather than the equations of motion of the $\mathrm{SU}(2)$ gauge theory.

\section{Ginzburg - Landau Lagrangian}

We now want to show that an effective, complex, Higgs-like, scalar field can be obtained from the $\mathrm{SU}(2) / \mathrm{U}(1)$ coset part of the $\mathrm{SU}(2)$ gauge theory. The self interaction of this effective scalar field is a consequence of nonlinear terms in the original Yang-Mills Lagrangian. The mass term for the scalar field is generated via the condensation of ghosts fields as discussed in the following section.

Making a connection between scalar and gauge fields is not a new idea. For

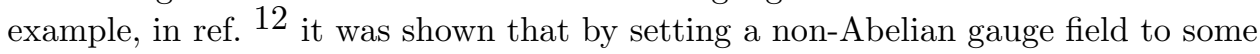
combination of a scalar field and its derivatives it was possible to obtain massless $\lambda \varphi^{4}$ theory. One could obtain a massive $\lambda \varphi^{4}$ theory by starting with Yang-Mills theory, but the mass term had to be inserted by hand 13. The final scalar field Lagrangian that we obtain is also a massless $\lambda \varphi^{4}$ theory with the addition of a coupling to a $\mathrm{U}(1)$ gauge field. This is (except for the $\mathrm{U}(1)$ gauge field coupling)

similar to the result of 12 . In the present paper we exchange the two gauge field of the $\mathrm{SU}(2) / \mathrm{U}(1)$ coset for a complex scalar field, whereas in refs. 1213 one gauge field is exchanged for a real scalar field. We begin by taking the expectation of the SU(2) Lagrangian (the gauge fixing and Faddeev-Popov terms are dealt with in the next section)

$$
-\frac{1}{4}\left\langle Q\left|\mathcal{F}_{\mu \nu}^{\mathcal{A}} \mathcal{F}^{\mathcal{A} \mu \nu}\right| Q\right\rangle=-\frac{1}{4}\left\langle\mathcal{F}_{\mu \nu}^{\mathcal{A}} \mathcal{F}^{\mathcal{A} \mu \nu}\right\rangle=-\frac{1}{4}\left\langle\mathcal{F}_{\mu \nu}^{3} \mathcal{F}_{\mu \nu}^{3}+\mathcal{F}_{\mu \nu}^{m} \mathcal{F}_{\mu \nu}^{m}\right\rangle
$$

First we consider the term

$$
\left\langle\mathcal{F}_{\mu \nu}^{3} \mathcal{F}_{\mathcal{A} \mu \nu}^{3}\right\rangle=\left\langle\left(f_{\mu \nu}+\Phi_{\mu \nu}\right)\left(f^{\mu \nu}+\Phi^{\mu \nu}\right)\right\rangle
$$

where the field $f_{\mu \nu}$ is in the ordered phase and $\Phi_{\mu \nu}$ is in the disordered phase. From the second assumption of the previous section $a_{\mu}$ and $f_{\mu \nu}=\partial_{\mu} a_{\nu}-\partial_{\nu} a_{\mu}$, behave as classical fields so that

$$
\left\langle f_{\mu \nu} \Phi^{\mu \nu}\right\rangle=f_{\mu \nu}\left\langle\Phi^{\mu \nu}\right\rangle, \quad\left\langle f_{\mu \nu} f^{\mu \nu}\right\rangle=f_{\mu \nu} f^{\mu \nu}, \quad\left\langle\Phi_{\mu \nu}\right\rangle=g \epsilon^{3 m n}\left\langle A_{\mu}^{m} A_{\nu}^{n}\right\rangle
$$


For the expectation of $A_{\mu}^{m}(y) A_{\nu}^{n}(x)$ we take the form

$$
\left\langle A_{\mu}^{m}(y) A_{\nu}^{n}(x)\right\rangle=-\delta^{m n} \eta_{\mu \nu} \mathcal{G}(y, x)
$$

$\mathcal{G}(y, x)$ is an arbitrary function. We note that strictly speaking this form of the expectation is an approximation since it reduces the degrees of freedom of the initial set of coset gauge fields, $A_{\mu}^{m}$ (this is our purpose - to find some approximate description of non-perturbative quantization). Nevertheless these degrees of freedom should be quantized at the next level of approximation. We hope that they can be quantized by perturbative Feynman diagram techniques.

The form given in (13) is consistent with the color and Lorentz indices of the left hand side. One might think to add a term with an index structure like $\eta_{\mu \nu} \epsilon^{3 m n}$. However, this is antisymmetric under exchange of $A_{\mu}^{m}(y) A_{\nu}^{n}(x)$ (i.e. exchanging both Lorentz and group indices) which is not consistent with the bosonic statistics of the gauge fields. The quantity in (13) is a mass dimension 2 condensate. The role of such gauge non-invariant quantities in the Yang-Mills vacuum has been discussed by several authors 1514 .

From (13) we find

$$
\begin{gathered}
\left\langle\Phi_{\mu \nu}\right\rangle=0 \\
\left\langle\Phi_{\mu \nu} \Phi^{\mu \nu}\right\rangle=g^{2}\left(\left\langle A_{\mu}^{1} A_{\nu}^{2} A^{1 \mu} A^{2 \nu}\right\rangle+\left\langle A_{\mu}^{2} A_{\nu}^{1} A^{2 \mu} A^{1 \nu}\right\rangle\right. \\
\left.-\left\langle A_{\mu}^{2} A_{\nu}^{1} A^{1 \mu} A^{2 \nu}\right\rangle-\left\langle A_{\mu}^{1} A_{\nu}^{2} A^{2 \mu} A^{1 \nu}\right\rangle\right) .
\end{gathered}
$$

Approximating these quartic gauge field expectation terms as sums of products of quadratic gauge field expectations (e.g. sums of terms like $\delta^{m p} \delta^{n q} \eta_{\alpha \mu} \eta_{\beta \nu} \mathcal{G}\left(x_{1}, x_{3}\right) \mathcal{G}\left(x_{2}, x_{4}\right)$ and symmetric permutation of all indices and coordinates. See ref. 11 for details) one finds that (15) becomes

$$
\left\langle\Phi_{\mu \nu}(x) \Phi^{\mu \nu}(x)\right\rangle \approx 24 g^{2} \mathcal{G}^{2}(x, x)
$$

The next term from (10) is

$$
\left.\left\langle\mathcal{F}_{\mu \nu}^{m}(y) \mathcal{F}^{m \mu \nu}(x)\right\rangle\right|_{y=x}
$$

The details of the evaluation of this expression are given in 11] The evaluation of (17) involves terms like

$$
\left.\left\langle\left[\partial_{\mu y} A_{\nu}^{m}(y)\right]\left[g \epsilon^{3 m n} a^{\mu}(x) A^{n \nu}(x)\right]\right\rangle\right|_{y=x}=\left.g \epsilon^{3 m n} a^{\mu}(x)\left\langle\left[\partial_{\mu y} A_{\nu}^{m}(y)\right] A^{n \nu}(x)\right\rangle\right|_{y=x}
$$

which require a bit of further explanation. In eq. (13) we excluded terms proportional to $\eta_{\mu \nu} \epsilon^{3 m n}$ because of the Bose symmetry of the gauge fields. In the expression in (18) the gauge fields do not appear symmetrically so such a term can be included. Thus we take the expectation of (18) to have the general form

$$
\left\langle\left[\partial_{\mu y} A_{\alpha}^{m}(y)\right] A_{\beta}^{n}\right\rangle=-\delta^{m n} \eta_{\alpha \beta} \partial_{\mu y} \mathcal{G}(y, x)-i \epsilon^{3 m n} \eta_{\alpha \beta} \partial_{\mu y} \mathcal{P}(y, x) .
$$


where $\mathcal{P}(y, x)$ is some general function. This new term will mix gauge bosons of different colors. Using the ansatz in (19) and (13) (18) becomes 11

$$
\begin{aligned}
\left\langle\mathcal{F}_{\mu \nu}^{m} \mathcal{F}^{m \mu \nu}\right\rangle & =-20\left[\partial_{\mu y} \partial_{x}^{\mu} \mathcal{G}(y, x)+g^{2} a_{\mu}(x) a^{\mu}(x) \mathcal{G}(x, x)\right. \\
& \left.-i g a^{\mu}(x) \partial_{\mu x} \mathcal{P}^{*}(y, x)+i g a^{\mu}(x) \partial_{\mu x} \mathcal{P}(y, x)\right]\left.\right|_{y=x}
\end{aligned}
$$

We now make the approximation that both functions, $\mathcal{G}(y, x)$ and $\mathcal{P}(y, x)$, can be rewritten in terms of a single complex scalar function as

$$
\mathcal{G}(y, x)=\mathcal{P}(y, x)=\frac{1}{5} \varphi^{*}(y) \varphi(x)
$$

Setting both $\mathcal{G}(y, x)$ and $\mathcal{P}(y, x)$ equal to the same product of a complex scalar field is called the one function approximation or ansatz. The factor of $1 / 5$ is to ensure that the kinetic term of this scalar field will have a factor of 1 in front of it. Using (21) in (20) we find

$$
\left\langle\mathcal{F}_{\mu \nu}^{m} \mathcal{F}^{m \mu \nu}\right\rangle=-4\left|\partial_{\mu} \varphi-i g a_{\mu} \varphi\right|^{2} \equiv-4\left(D_{\mu} \varphi^{*}\right)\left(D^{\mu} \varphi\right) .
$$

Thus the total Lagrangian density is

$$
\langle\mathcal{L}\rangle=-\frac{1}{4}\left\langle\mathcal{F}^{\mathcal{A}}{ }_{\mu \nu} \mathcal{F}^{\mathcal{A} \mu \nu}\right\rangle=-\frac{1}{4} f_{\mu \nu} f^{\mu \nu}+\left(D_{\mu} \varphi^{*}\right)\left(D^{\mu} \varphi\right)-\frac{6 g^{2}}{25}|\varphi|^{4}
$$

This is the GL Lagrangian, with a massless, effective scalar field. This scalar field is connected with the off diagonal gauge fields by (13) (21). This lack of a mass term is a shortcoming for the effective Lagrangian of (23). Without it there is no spontaneous symmetry breaking and no Nielsen-Olesen flux tube solutions, both which are critical to make the connection to the dual superconducting picture of the QCD vacuum. In the next section we show how a condensation of ghosts fields can lead to a mass term for the effective scalar field $\varphi$. This mass term is of the correct form (i.e. tachyonic) to give rise to spontaneous symmetry breaking and Nielsen-Olesen flux tube solutions.

\section{Tachyonic mass term via ghost condensation}

In ref. 3 (see also 4 ) it was shown that a tachyonic mass term is generated for the off-diagonal gauge fields of a pure SU(2) Yang-Mills via a condensation of ghost and anti-ghost fields. To the Lagrangian of (18) one adds gauge fixing and FaddeevPopov terms of the form

$$
\begin{aligned}
\mathcal{L}_{G F}+\mathcal{L}_{F P} & =-\frac{1}{2 \alpha}\left(D_{\mu}^{m n} A^{\mu n}\right)^{2}+i \bar{C}^{m} D_{\mu}^{m p} D^{\mu p n} C^{n} \\
& -i g^{2} \epsilon^{m q} \epsilon^{p n} \bar{C}^{m} C^{n} A^{\mu p} A_{\mu}^{q}+\frac{\alpha}{4} g^{2} \epsilon^{m n} \epsilon^{p q} \bar{C}^{m} \bar{C}^{n} C^{p} C^{q}
\end{aligned}
$$

where $\epsilon^{m n}$ is the antisymmetric symbol for the off-diagonal indices $\left(\epsilon^{12}=-\epsilon^{21}=1\right.$ and $\left.\epsilon^{11}=\epsilon^{22}=0\right)$, and $D_{\mu}^{m n}=\partial_{\mu} \delta^{m n}-g \epsilon^{m n} a_{\mu}$. Next, replacing the quartic ghost 
interaction (the last term in (24)) with an auxiliary field, $\psi, 34$ and applying the Coleman-Weinberg mechanism 15 one finds that a ghost condensation occurs with

$$
\left\langle i \bar{C}^{m} C^{m}\right\rangle=-\frac{v}{16 \pi}<0
$$

since $v>0$. The third term in the Lagrangian in (24) now becomes

$$
i \epsilon^{m q} \epsilon^{p n} \bar{C}^{m} C^{n} A^{\mu p} A_{\mu}^{q} \rightarrow \frac{1}{2}\left\langle i \bar{C}^{m} C^{m}\right\rangle\left\langle A_{\mu}^{n} A^{\mu n}\right\rangle=\frac{1}{2}\left(\frac{v}{16 \pi}\right)(8 \mathcal{G})=\frac{v}{20 \pi} \varphi^{*} \varphi
$$

where (13) and (21) have been used. Putting this term together with the quartic term from the effective GL Lagrangian in (23) we find that $\varphi$ has developed an effective potential of the form

$$
V_{\varphi}=-\frac{v g^{2}}{20 \pi}|\varphi|^{2}+\frac{6 g^{2}}{25}|\varphi|^{4}
$$

A tachyonic mass term $\left(m^{2}=-\left(v g^{2}\right) /(20 \pi)<0\right)$ has been generated for the effective scalar field, $\varphi$, with the consequence that spontaneous symmetry breaking occurs. We have arrived at an effective massive GL-Lagrangian with the tachyonic mass term for the $\varphi$ field being generated by ghost condensation. This effective GLLagrangian will also have Nielsen-Olesen flux tube solutions. With this tachyonic mass term for $\varphi$ spontaneous symmetry breaking occurs and the $U(1)$ field, $a^{\mu}$, will develop a mass of $\sqrt{\left(5 g^{2} v\right) /(24 \pi)}$. It can be shown 34 that the ghost condensate will not give a contribution to the mass of $a^{\mu}$, via the term $i \bar{C}^{a} C^{a} a_{\mu} a^{\mu}$.

\section{Conclusions}

In this paper we have combined several ideas (Abelian projection, quantization methods originally proposed by Heisenberg, and some assumptions about the forms of various expectation values of the gauge fields) to show that one can construct an effective scalar field within a pure $\mathrm{SU}(2)$ gauge field theory. The system of effective scalar field plus the remaining Abelian field is essentially scalar electrodynamics or the relativisitic version of the GL Lagrangian. There is also a connection between the present work and Cho's 16 "magnetic symmetry" study of the dual Meissner effect within Yang-Mills theory. In refs. 16] a Lagrangian similar to our eq. (23) is obtained, with the complex scalar field being associated with a monopole coupled to a U(1) dual magnetic gauge boson. This may offer one possible physical interpretation of our scalar field of eq. (21): it may represent some effective monopole-like field which results from the $\mathrm{SU}(2) / \mathrm{U}(1)$ coset fields. This is in accord with lattice QCD simulations which indicate that monopole condensation plays a role in color confinement 17. in ref. 18 very similar idea is proposed: the reduction from the $\mathrm{SU}(2)$ Yang-Mills theory to a two-band dual superconductor with an interband Josephson coupling.

The essential physics here is that one has disordered fields (the gauge fields of the $\mathrm{SU}(2) / \mathrm{U}(1)$ coset space or equivalently the effective, complex, scalar field) which "pushes out" (i.e. exhibits the Meissner effect) the ordered field (the Abelian, 
U(1) field) except in the interior of the flux tubes. This is a continuation of ref. 1 which supports the dual superconducting picture of the Yang-Mills vacuum for a pure gauge field. The scalar field comes from some subset of the gauge fields rather than being put in by hand. An interesting question is if the procedure in this paper can be applied to the $\mathrm{SU}(3)$ gauge theory of the strong interaction 19 . In ref. $\underline{6}$ the $\mathrm{SU}(3)$ Lagrangian with quarks was studied, and using a procedure similar to the one in the present paper, it was found that the dual Meissner effect did occur.

\section{Acknowledgment}

VD is grateful Prof. H. Kleinert for invitation for the research and Alexander von Humboldt Foundation for the support of this work.

\section{References}

1. V. Dzhunushaliev and D. Singleton, Phys. Rev., D65, 125007 (2002).

2. H.B. Nielsen and P. Olesen, Nucl. Phys. B61, 45 (1973)

3. D. Dudal and H. Verschelde, J.Phys. A36, (8507) 2003.

4. V.E.R. Lemes, M.S. Sarandy, and S.P. Sorella, J.Phys., A36, (7211) 2003.

5. Kei-Ichi Kondo, Phys. Lett. B514, 335 (2001); "Dual superconductivity, monopole condensation and confining string in low-energy Yang-Mills theory. Part I", hep-th0009152

6. R. Parthasarathy, Mod. Phys. Lett. A 15, 2037 (2000)

7. Kei-Ichi Kondo, Phys. Rev. D57, 7467 (1998)

8. W. Heisenberg, Introduction to the unified field theory of elementary particles., Max - Planck - Institut für Physik und Astrophysik, Interscience Publishers London, New York, Sydney, 1966; W. Heisenberg, Nachr. Akad. Wiss. Göttingen, N8, 111(1953); W. Heisenberg, Zs. Naturforsch., 9a, 292(1954); W. Heisenberg, F. Kortel und H. Mütter, Zs. Naturforsch., 10a, 425(1955); W. Heisenberg, Zs. für Phys., 144, 1(1956); P. Askali and W. Heisenberg, Zs. Naturforsch., 12a, 177(1957); W. Heisenberg, Nucl. Phys., 4, 532(1957); W. Heisenberg, Rev. Mod. Phys., 29, 269(1957).

9. A. Di Giacomo, H. G. Dosch, V. I. Shevchenko, Yu. A. Simonov, "Field correlators in QCD. Theory and applications", hep-ph/0007223

10. Yu. A. Simonov, "Self coupled equations for the field correlators", hep-ph/9712250

11. V. Dzhunushaliev and D. Singleton, Mod. Phys. Lett. A, 18, 955 (2003).

12. G. 't Hooft, Phys. Rev. Lett., 37, 8 (1976); E. Corrigan and D. Fairlie, Phys. Lett., B67, 69 (1977); F. Wilczek, in Quarks Confinement and Field Theory, (ed. D. Stump and D. Weingarten, Wiley, New York 1977)

13. A. Actor, Lett. Math. Phys., 2, 275 (1978)

14. F.V. Gubarev, L. Stodolsky, and V.I. Zakharov, Phys. Rev. Lett., 86, 2220 (2001); F.V. Gubarev and V.I. Zakharov, Phys. Lett. B501, 28 (2001)

15. S. Coleman and E. Weinberg, Phys. Rev., D7, 1888 (1973).

16. Y.M. Cho, Phys. Rev. D21, 1980; Phys. Rev. D23, 1981

17. A. Kronfeld, G. Schierholz and U.-J. Wiese, Nucl. Phys. B293, 461 (1987); A. Kronfeld, M. Laursen, G. Schierholz and U.-J. Wiese, Phys. Lett. B198, 516 (1987)

18. Antti J. Niemi, JHEP, 0408, 035 (2004); "Dual Superconductors and SU(2) YangMills, hep-th/0403175

19. V. Dzhunushaliev, D. Singleton and T. Nikulicheva, "Nonperturbative calculational method in quantum field theory," hep-ph/0402205 V. Dzhunushaliev and D. Singleton, Mod. Phys. Lett. A, 18, 2873 (2003) 